\title{
Kommen Kollokationen in Mode? Kollokationskonzepte und ihre mögliche Umsetzung in der Didaktik
}

\author{
Anna Reder (Pécs)
}

\begin{abstract}
Collocation is defined differently in the various linguistic disciplines and no generally acceptable definition exists. One reason for that is the variety of approaches and another is the complexity of the phenomenon. It follows from the semantic concept of collocation for language learning that the acquisition of vocabulary means the learning of words in isolation and the properties which determine their compatibility with other words. This is an inefficient way and often involves interference errors. In the interpretation of corpus linguistics the learning of words occurring together is encouraged. The student is able to find the collocator matching the basis in a corpus. Searching a corpus, however, has both advantages (learning collocations by examples) and disadvantages (disregarding less frequent collocations). The paper argues that collocations should be conceived as phrasal units and taught using a threephase model. The target is to achieve psycholinguistic fixedness in the mental lexica of students through effective exercises.
\end{abstract}

\section{$1 \quad$ Das Interesse an Kollokationen}

Die Fragestellung in der Überschrift impliziert die Annahme, dass das Interesse an Kollokationen kontinuierlich zunehmen wird. Ist das noch überhaupt eine aktuelle Erwartung? Ist ein Diskurs über Kollokationen nicht längst schon gang und gäbe? Wenn man in der Fachliteratur recherchiert, findet man tatsächlich zahlreiche einschlägige Publikationen. Schon in den 80er Jahren des vergangenen Jahrhunderts behandelt Hausmann (1984) Kollokationen und legt dem Leser nahe, dass Wortschatzlernen Kollokationslernen sei. Seitdem erscheinen immer wieder Studien über Kollokationen. Um die Jahrtausendwende ist sogar ein deutlicher Zuwachs an Veröffentlichungen zu verzeichnen (cf. z. B. Dörr 2005; Hausmann 2007; Hollós 2004; Lütge 2002; Reder 2006; Scherfer 2001; Siepmann 2003; Steinbügel 2005). Eine beliebige Auswahl der einschlägigen Fachliteratur belegt also, dass Kollokationen bereits in mehreren Disziplinen, wie Lexikologie, Lexikographie, Fachsprachenforschung, Korpuslinguistik und Sprachlehrforschung, intensiv erforscht werden. Kollokationen sind also in Mode, könnte man schlussfolgern, wenn man ausschließlich die Forschung betrachtet. Mit einem Blick auf die Unterrichtspraxis stellt sich jedoch die Frage: Wie verhält es sich mit der Umsetzung der Forschungsergebnisse in der Didaktik? Vorliegender Aufsatz geht dementsprechend folgenden Fragestellungen nach: Welche Vorteile und Nachteile hat die Anwendung einzelner Kollokationskonzepte in der Unterrichtspraxis? Welchen Raum lassen Lehrwerke der Entwicklung des Kollokationsbewusstseins von Lernern zu? 


\section{Kollokationsdeutungen und ihre Anwendung}

Ein relevanter Störfaktor in Bezug auf die Umsetzung der Erkenntnisse der Kollokationsforschung in der Unterrichtspraxis ist gleich vorwegzunehmen. Eine einheitliche Kollokationsdefinition ist ein ungelöstes Forschungsproblem. Wichtige Faktoren für die Definitionsschwierigkeit sind zum einen die Komplexität des Phänomens und zum anderen die unterschiedlichen Herangehensweisen. So hat der Kollokationsbegriff in der einschlägigen Literatur mehrere Deutungen. Je nach Perspektiven der einzelnen Disziplinen kommen wir zu unterschiedlichen Auslegungen. Im Folgenden werden einige Definitionen und ihre Umsetzungsmöglichkeiten in der Didaktik angesprochen.

\subsection{Kollokation als Kompatibilität der Wörter. Einzelne Wörter als Bausteine von Lerneräußerungen}

In der Semantik versteht man unter Kollokation einen abstrakten Begriff, nämlich die Kompatibilität, die semantische Verträglichkeit der Wörter miteinander. Im Satz

Studien belegen, "dass falsche Lernmethoden zu Blockaden im Gehirn führen können".1

sehen wir, dass im Hauptsatz die Autosemantika Studien und belegen verträglich sind. Im Nebensatz stehen falsche und Lernmethoden nebeneinander, also auch sie "vertragen sich". Das Verb mit seiner Rektion führen $+z u$ ist kompatibel mit Blockaden und dieses Substantiv "toleriert" die Nominalphrase im Gehirn neben sich.

Wenn wir den abstrakten Kollokationsbegriff, der die Relation von Wörtern erfasst, im Lernprozess anwenden, sind wir mit einem bedeutenden Problem konfrontiert. Diese Begriffsdeutung ist nämlich wortorientiert. Sie legt dem Lerner nahe, dass die Äußerungen aus isolierten Wörtern aufgebaut werden. Man sucht sich Wörter aus, die semantisch verträglich sind und reiht sie nebeneinander. Das scheint plausibel zu sein. Die Falle ist jedoch, dass die semantische Verträglichkeit der Wörter häufig sprachspezifisch ist. Das, was in der einen Sprache üblich ist, kann in der anderen ungewöhnlich klingen. Im Deutschen sind z. B. Höchstleistungen und erbringen kompatibel, während im Ungarischen Höchstleistungen mit anbieten verträglich ist (ung: csúcsteljesítményt nyújt). In anderen Fällen ist wiederum die Verträglichkeit übertragbar. Für dieses Phänomen sind Ordnung halten und seine ungarische Entsprechung rendet tart ein gutes Beispiel.

Die semantische Kollokationsauffassung hat also für den Lernprozess zwei negative Folgen. Zum einen ist es bekanntlich nicht ökonomisch einzelne Wörter als Bausteine zu nehmen. Aus wortübergreifenden Einheiten lässt sich wohl ein Satz schneller formulieren. Zum anderen hat sich der Lerner über die Wortform sowie die denotative und eventuelle konnotative Bedeutung hinaus noch die kollokable Bedeutung einzelner Wörter anzueignen. Er muss sich auch merken, ob die Kompatibilität in der Fremdsprache äquivalent mit der in der Muttersprache ist. Dies erfordert jedoch einen enormen kognitiven Aufwand, der nicht zwangsläufig zur normgerechten Sprachproduktion führt. Denn in Lernertexten stößt man immer wieder auf eine falsche Wortwahl, die vermutlich auf einen Bedeutungstransfer der kollokablen Bedeutung eines Wortes zurückzuführen ist. Das ist auch in der folgenden typischen Lerneräußerung ungarischer Germanistikstudenten in Hausarbeiten zur Phraseologie der Fall: *In Texten trifft man sich häufig mit Idiomen. Der Lerner berücksichtigt im obigen Satz die Beschränkung des Verbs sich treffen in der Bedeutung 'zusammenkommen mit anderen' nicht auf Personen, da diese Verbindbarkeitsregel für ihn auf der Grundlage der L1 völlig fremd ist. Das Fehlerbeispiel belegt, dass er sich der Kompatibilitätsunterschiede zwischen dem Verb sich treffen und seinem ungarischen Äquivalent nicht bewusst ist. Es ist also davon auszugehen, dass der Lerner das Verb sich

\footnotetext{
${ }^{1}$ www.mytopic.at/home/schule-job/lerntipps/60, Stand 15.05.2010.
} 
treffen als isoliertes Wort erlernt hat und die ungarischen Verträglichkeitsrelationen überträgt. Das Ungarische differenziert nämlich nicht, ob man sich mit Personen trifft oder auf etwas trifft. Es ist anzunehmen, dass die regelhafte Verwendung der Verben sich treffen $($ mit $+D)$ und treffen $(a u f+A)$ dadurch gefördert werden kann, wenn sich der Lerner diese nicht als isolierte Verben erwirbt. Der Unterdifferenzierung wirkt es vermutlich entgegen, wenn der Lerner frequente Wortverbindung, wie z. B. sich mit Freunden (Personen) treffen, in Texten auf Idiome treffen als Einheiten internalisiert.

\subsection{Kollokationen als frequente Wortkombinationen. Häufige Wortverbindungen als Bausteine von Lerneräußerungen}

Im obigen Abschnitt wird eindeutig gegen die Anwendung der semantischen Kollokationsdefinition im Unterricht argumentiert, da dieser abstrakte Begriff zum Wortschatzlernen auf der Wortebene führt. Der semantische Begriff Kompatibilität ist für den Lernprozess auch deshalb nicht einfach zu operationalisieren, weil in Texten nicht nur übliche Mehrwortverbindungen wie z. B. allein erziehende Mutter, sondern auch kreative Wortkombinationen vorkommen wie etwa allein verziehende Mutter im folgenden Text:

Ich habe derzeit ziemlichen Zoff und kann mit niemandem darüber reden. Meine allein verziehende Mutter sehe ich in der Früh immer nur fünf Minuten. Am Abend kommt sie in letzter Zeit sehr spät heim, ist dann hundemüde und will ihre Ruhe. ${ }^{2}$

Übliche Wortverbindungen fungieren als guter Input zur Darbietung der Kompatibilitätsregel des jeweiligen Wortes. Okkasionelle Verwendungen, die praktisch gegen Kompatibilitätsregeln verstoßen, sind jedoch nicht geeignet, um auf Verträglichkeitsbeziehungen $\mathrm{zu}$ schließen. So sind gelegentliche Wortkombinationen als Elemente des Verstehenswortschatzes zu betrachten und es ist diesen nach erfolgreicher Rezeption keine weitere Aufmerksamkeit mehr zu schenken.

Wie kann jedoch der Sprachlerner oder der nicht muttersprachliche Lehrende entscheiden, ob eine Wortkombination gebräuchlich ist und eventuell zum aktiven Sprachgebrauch internalisiert werden soll? Bei der Entscheidung, ob eine Wortverbindung okkasionell oder üblich ist, schafft die kontextualistische Herangehensweise Abhilfe. Im Kontextualismus deutet man den Kollokationsbegriff unter dem Aspekt der Frequenz. Wörter, die in Texten signifikant häufig miteinander auftreten, bilden Kollokationen. Überprüfen wir auf dem Wortschatzportal Leipzig ${ }^{3}$, mit welchen Wörtern z. B. das Verb belegen häufig vorkommt, ergibt die Korpussuche, dass Studien zu seinen häufigsten Partnern gehört (1159). Aus der Gegenprobe, bei der Studien als Lemma angegeben wird, geht hervor, dass belegen im unmittelbaren Satzkontext von Studien sehr häufig anzutreffen ist. Der kontextualistische Kollokationsbegriff stellt also Kookkurrenzen in den Mittelpunkt. Kontextualisten "gehen vom statistischen Ansatz aus und verstehen unter der Kollokation eines Wortes die Wörter, die signifikant häufig mit diesem Wort [...] erscheinen". 4

Das Umsetzen dieser Kollokationsauffassung in der Unterrichtspraxis bringt den Vorteil, dass Lerner auch autonom in digitalen Korpora Kollokationen ausmachen können. Die Frequenzuntersuchung zu Termine ergibt z. B., dass das Verb eintragen signifikant häufig im Kontext erscheint. So können die Lerner frequente Wortverbindungen aus digitalen Korpora für den eigenen Gebrauchswortschatz extrahieren.

\footnotetext{
2 Dani \& Didi in Jugend Österreich März 2005: lehrerservice.at/joarchiv/display.php?ausgabe=ag-0407\&artikel=A04-07-06.htm, Stand 15.05.2010.

3 wortschatz.uni-leipzig.de, Stand 8. November 2010.

${ }^{4}$ wortschatz.uni-leipzig.de/html/faq/koloka.html, Stand 15.05.2010.
} 
Auch beim Erstellen von schriftlichen Produkten können Kollokationsangaben digitaler Korpora passende Kollokatoren liefern. Wie dabei das Wortschatzportal der Universität Leipzig mit den Kollokationsinformationen die Benutzer unterstützen kann, soll exemplarisch an der folgenden Lerneräußerung gezeigt werden:

Ich habe die Absicht, Strategien beim Vokabellernen auszuprobieren.

Diese Äußerung ist zweifelsohne verständlich und grammatisch korrekt, drückt jedoch die Sprechabsicht des Lerners nicht vollkommen aus, wenn er auch darüber sprechen will, dass seine Intention stark ist. So kann dieser Satz mit Hilfe der Kollokationsangaben des Wortschatzportals vom Lerner selbst optimiert werden. Zum Lemma Absicht werden unter den signifikanten linken Nachbarn folgende häufige Partnerwörter angeführt:

"Signifikante linke Nachbarn von Absicht:

die (5189), seine (1015), keine (697), ihre (686), betrügerischer (663), mit (448), voller (379), feste (369), böse (363), meine (319), Die (300), böser (238), der (230), [...]"

(http://wortschatz.uni-leipzig.del $\rightarrow$ "Absicht", Stand 15.05.2010)

Unter den möglichen Kollokatoren rangiert das passende Adjektiv fest relativ vorne. So kommt der Lerner mit Hilfe vom Wortschatzportal auf die folgende verbesserte Version:

Ich habe die feste Absicht, Strategien beim Vokabellernen auszuprobieren.

Die statistische Häufigkeit und ihre automatische Ermittlung können den Lerner also beim Erkennen und Bilden von Kollokationen im kontextualistischen Sinne unterstützen.

Ist das jedoch immer der Fall? Haben wir mit den digitalen Korpora ein effektives Hilfsmittel für Fremdsprachenlerner beim Kollokationsgebrauch gefunden? Zum Teil schon, denn einige Versuche in der Praxis zeigen, dass fortgeschrittene Deutschlerner effektiv mit dem Kollokatorangebot umgehen können. Die im Folgenden besprochenen Beispiele, die den effektiven Umgang mit Kollokationen im Unterricht illustrieren sollen, gehen auf ein Lexikographieseminar zurück, das ich im Sommersemester 2009/2010 an der Universität Pécs unterrichtet habe.

Zwei Schwierigkeiten sind hier jedoch anzusprechen. Erstens kann im obigen Beispiel der Lerner seine Aussage erst dann intensivieren, wenn er das entsprechende Wort unter den Kollokatorangaben mit Hilfe seiner Sprachkompetenz wiedererkennt. Ohne entsprechende Wortschatzkenntnisse überfordert ihn die lange Liste der möglichen Kollokatoren. Es ist aber auch erforderlich, dass der Lerner mit der Suchstrategie auf dem Wortschatzportal vertraut ist, denn bei der automatischen Suche werden nicht nur Autosemantika, sondern auch Synsemantika ermittelt. Da diese jedoch in Bezug auf die Aufgabe - Intensivierung von Absicht - nicht relevant sind, müssen die Lerner sie überlesen.

Die zweite Schwierigkeit bei der Kollokatorsuche auf dem Wortschatzportal ergibt sich für den Lerner dadurch, dass die automatische Frequenzuntersuchung nur einzelne Wortformen berücksichtigt und außerdem die Bedeutungsvarianten des jeweiligen Lemmas nicht auseinander halten vermag. Der Lerner hat jedoch einen konkreten Kontext, in dem das Lemma monosemiert ist. Nehmen wir zur Veranschaulichung dieses Problems erneut das obige Beispiel die Absicht haben. Das Verb haben bildet einen weiteren Kontext, der auch eine grammatische Restriktion impliziert. Deshalb kommt nur eine Akkusativergänzung in Frage. So scheidet der Kollokatorkandidat voller eindeutig aus, obwohl er einen Platz vor feste rangiert. Aber er steht nicht im Akkusativ, denn er ist ein Teil der Kombination mit voller Absicht. Am Ende der Liste wird zwar volle grammatisch passend angeführt, aber die Position zeigt keine hohe Frequenz (volle 24). Bei der Auswahl des üblichen Adjektivs 
braucht also der Lerner nicht nur semantisches Wissen zum Wiedererkennen des Wortes, sondern hat auch den jeweiligen Kontext zu berücksichtigen.

Das Beispiel feste Absicht zeigt exemplarisch, dass die Kollokatorsuche für AdjektivSubstantiv-Kollokationen auf dem Wortschatzportal erfolgversprechend ist, da die Basis und der Kollokator unmittelbare Nachbarn sind. Bei anderen Strukturtypen, in denen die Bestandteile nicht unmittelbar aufeinander folgen, ist die Kollokatorsuche auf dem Wortschatzportal für Fremdsprachenlerner etwas mühsamer. Dann sind nämlich die entsprechenden Partnerwörter im "Dschungel" der signifikanten Kookkurrenzen zu finden.

Es gibt auch Suchfragen, bei denen das Wortschatzportal überfragt ist. Ein wichtiger Grund dafür ist, dass die automatisch ermittelten Kollokationen weitgehend davon abhängen, welche Texte im Korpus aufgenommen worden sind. Wenn wir z. B. nach einem entsprechenden Verb zu Lernstrategien suchen oder uns vergewissern wollen, ob die Verben einsetzen, anwenden oder gebrauchen in Verbindung mit Lernstrategien üblich sind, ermittelt das Wortschatzportal keine passenden signifikanten Kookkurrenzen. Im für das Wortschatzportal vorliegenden Korpus kommt nämlich das Wort nur selten vor. Auch die Eingabe der Wortform Strategien in die Suchmaske des Wortschatzportals hilft nicht weiter. Die Verben einsetzen, anwenden oder gebrauchen werden nicht angeführt, so bekommt man bei der Recherche keine Bestätigung für diese Kollokatorkandidaten. Ein weiteres Suchen in Texten, die uns Suchmaschinen zur Verfügung stellen, ist erforderlich, um Belege für das Miteinandervorkommen von Lernstrategien und den obigen Verben zu finden. Eine weitere digitale Suchmöglichkeit bieten die öffentlichen Korpora des Instituts für deutsche Sprache5.

\subsection{Kollokationen als Phraseme. Feste Wortverbindungen als Bausteine von Lerneräußerungen}

Die kontextualistische Kollokationsbestimmung, das frequente Miteinandervorkommen einzelner Wörter, wird - wie oben exemplarisch beschrieben - z.B. auf dem Wortschatzportal der Universität Leipzig automatisch an Texten durchgeführt. So bietet diese Seite bei der Suche nach Kollokatoren auch Deutschlernern ein mehr oder weniger effektives Werkzeug.

Kommen wir noch mal auf das Beispiel Lernstrategien anwenden / verwenden / einsetzen / gebrauchen zurück. Nehmen wir Lernstrategien als Suchwort, so gibt das Wortschatzportal ${ }^{6}$ anwenden nicht als Kollokator an. Die Suche nach den "Partnern" einzelner Wörter ist also nicht in jedem Einzelfall erfolgreich. So bleibt uns nichts anderes übrig, als die Wortebene zu verlassen und Kollokationen als polylexikale Wortschatzeinheiten zu deuten. Das heißt, wir recherchieren nicht nach Kollokatoren zu der Basis Lernstrategien, sondern überprüfen die Häufigkeit folgender Wortverbindungen: Lernstrategien anwenden, Lernstrategien verwenden, Lernstrategien einsetzen und Lernstrategien gebrauchen. Wenn wir die Einheit Lernstrategien anwenden in einem digitalen Korpus, z. B. in Google in Anführungsstrichen eingeben, bekommen wir schon für die Nennform eine relativ hohe Trefferzahl, 1330. An der zweiten Stelle rangiert Lernstrategien einsetzen mit der Trefferzahl 333. Dem folgen Lernstrategien verwenden mit 177 Treffern und Lernstrategien gebrauchen mit 2 Treffern ${ }^{7}$. Somit können die letzten beiden als mögliche aber weniger übliche Wortverbindungen ausscheiden. Die Recherche in Suchmaschinen ist jedoch nicht unproblematisch. Die ermittelten Werte sind nämlich keine absoluten Zahlen sondern sie sind lediglich als Tendenzen (Konecny 2010: 84) zu betrachten. Auf relevante Probleme weisen auch Bubenhofer/Ptashnyk hin:

\footnotetext{
5 www.ids-mannheim.de/cosmas2/, Stand 8. November 2010.

${ }^{6}$ wortschatz.uni-leipzig.de, Stand 15. Mai 2010.

${ }^{7}$ www.google.de, Suche am 15. Mai 2010.
} 
Nachteile der Nutzung bestehender Suchmaschinen liegen einerseits darin, dass sie nicht für linguistische Recherchen gedacht sind und der Suchalgorithmus nicht im Detail bekannt ist. Andererseits kann die Datengrundlage schlecht kontrolliert werden. Die Suchmaschinenbetreiber schweigen sich über den Umfang des indizierten Korpus aus. Es ist deshalb nicht möglich, Frequenzen in Relation zur Korpusgröße zu setzen. Zudem verändert sich die Zusammensetzung des Korpus naturgemäß laufend. (Bubenhofer/Ptashnyk 2010: 16)

Man bekommt also zwangsläufig immer wieder andere Werte, wann auch immer man "Lernstrategien anwenden" im Google eingibt. Aber diese sind ständig hoch. Diese Zahlen relativieren sich jedoch, da ein und derselbe Text eventuell in verschiedenen Dokumenttypen im Internet erreichbar ist, so wird derselbe Text mehrfach durchsucht (cf. Konecny 2010: 83).

Für Lernstrategien anwenden belegen also digitale Korpora eine nicht unbedeutende Frequenz und damit gleichzeitig eine wichtige phraseologische Eigenschaft, die Festigkeit. Eine Art der Festigkeit ist nämlich die Gebräuchlichkeit (cf. Burger 2007: 16). In der Phraseologie versteht man unter Kollokationen feste, aber nicht idiomatische Wortverbindungen (cf. Burger 2007: 38). Das sind also Wortverbindungen, die nicht frei, aber kompositionell sind. Wie lässt sich jedoch die Eigenschaft Festigkeit von Kollokationen erfassen? Eine eben bereits angesprochene Möglichkeit ist, Wortverbindungen als fest zu bezeichnen, wenn sie gebräuchlich sind. Die Festigkeit einer Kollokation kann man also nachweisen, indem man ihre Gebräuchlichkeit statistisch untermauert, wie das im vorigen Abschnitt mit Strategien anwenden demonstriert wurde. Aus der phraseologischen Perspektive fragt man sich jedoch mit Recht, ob tatsächlich "alles, was irgendwie frequent ist, ein Phrasem sein" sollte (Donalies 2009: 13). Aus phraseologischer Sicht sind also Kollokationen von freien Wortverbindungen durch weitere Eigenschaften abzuheben. Diese sind in Anlehnung an Burger (2007: 17-25) die psycholinguistische und die strukturelle Festigkeit.

Die psycholinguistische Festigkeit der Kollokationen wird durch Reaktionszeitmessungen belegt. Experimente zeigen, dass Kollokationen von Muttersprachlern schneller erkannt werden als freie Wortverbindungen. Im mentalen Lexikon des Sprechers sind Kollokationen entweder als Einheiten gespeichert oder zwischen den Bestandteilen ist eine bevorzugte Verbindung ausgebaut (cf. Schönefeld 2001). Die psycholinguistische Festigkeit, also die Speicherung der Kollokation als eine Ganzheit, ist für das fremdsprachliche mentale Lexikon eines Lerners nicht typisch. Der Lerner hat nämlich Einheiten in seinem mentalen Lexikon für Kollokationen in L1 gespeichert. So hat er für die nichtäquivalenten zielsprachlichen Kollokationen neue mentale Repräsentationen zu bilden. Es ist also davon auszugehen, dass zielsprachliche Kollokationen, die in L1 nichtäquivalente Entsprechungen haben, mehr Lernaufwand benötigen. Daraus folgt, dass die Lerner je nach L1 oder weiteren Fremdsprachen unterschiedliche Lernschwierigkeiten mit Kollokationen haben. Ein Deutschlerner ungarischer Muttersprache "freut sich" z. B. über die Kollokation Ordnung halten, da ihre Entsprechung im Ungarischen wortwörtlich ist. Die Kollokation Struktur in etwas bringen ist für ihn jedoch fremd, da er in L1 daran gewöhnt ist, dieses Konzept durch ein einfaches Verb auszudrücken. Übungen, in denen unter kontrastivem Aspekt ausgesuchte Kollokationen geschult werden, sind erforderlich, um die fehlende fremdsprachliche psycholinguistische Festigkeit einer Kollokation im mentalen Lexikon des Lerners auszubauen.

Der zweite Festigkeitstyp der Phraseme, die strukturelle Festigkeit, meint Irregularitäten und Restriktionen auf der sprachlichen Ebene (cf. Burger 2007: 20-25). Irregularität ist für Kollokationen nicht typisch. Auch Restriktionen sind auf der morpho-syntaktischen Ebene nicht besonders kennzeichnend. Einschränkungen können jedoch auf der lexiko-semantischen Ebene auftreten. Gemeint ist die Nichtaustauschbarkeit des Kollokators durch ein Synomym. Im Strategievorschlag 


\section{Bringe Struktur in deinen Lernstoff ${ }^{8}$}

ist z. B. der Kollokator bringen nicht durch ein Synonym auszutauschen. Aber auch die lexikalisch-semantische Restriktion ist nicht in jedem Einzelfall eine bindende Eigenschaft der Kollokationen. Im folgenden Lerntipp gibt es ein passendes Beispiel für dieses Phänomen:

"Beginn mit leichten Übungen: Wie ein Motor braucht auch dein Gehirn eine Aufwärmphase, bis es Höchstleistungen bringt"9

Der Kollokator bringen kann durch erbringen ersetzt werden, ohne dass dadurch der Kollokationsstatus der Wortverbindungen verloren geht. Ähnlich verhält es sich mit der Kollokation Studien belegen, da belegen durch beweisen austauschbar ist, auch wenn Letzteres weniger häufig vorkommt (cf. das Ergebnis der Suche nach "Studien" im Wortschatzportal ${ }^{10} \rightarrow$ Studien belegen 979 , beweisen 96 ).

In der Phraseologie werden Kollokationen mit einer weiteren Eigenschaft verbunden, und zwar mit der Nichtidiomatizität, die sie von den Idiomen mehr oder weniger trennt und mit den freien Wortgefügen verbindet (cf. Hausmann 2007). Da Kollokationen kompositionell sind, ergibt sich ihre Gesamtbedeutung additiv aus den Bedeutungen der Bestandteile. So sind Kollokationen im Wortschatz zwischen freien und idiomatischen Wortverbindungen (mit fließenden Grenzen) angesiedelt (siehe Abbildung 1):

\begin{tabular}{|lllll|}
\hline freie Wortverbindung & $\rightarrow$ & Kollokationen & $\rightarrow$ & Idiome \\
\hline
\end{tabular}

\section{Abbildung 1: Idiomatizitätsgrad}

Wenn wir die phraseologische Kollokationsauffassung in der Unterrichtspraxis umsetzen, ergibt sich der Vorteil, dass wir Kollokationen als feste Einheiten behandeln und dadurch ihre Speicherung als Einheit im Lernprozess gewiss fördern. Das Substantiv Studien und das Verb belegen sollten also nicht einzeln vermittelt und angeeignet werden, sondern die Kollokation Studien belegen. Kollokationen als Chunks zu erlernen hat zwar zur Folge, dass der Bereich der anzueignenden polylexikalen Einheiten ausgeweitet wird, aber das Einprägen der Kollokationen als Einheiten im mentalen Lexikon wird dadurch vermutlich erleichtert. Besonders relevant ist der Umgang mit jenen Kollokationen als Einheiten, deren Entsprechungen in L1 nicht total äquivalent sind.

Eine Schwierigkeit besteht jedoch bei der Vermittlung der Kollokationen darin, dass nicht nur prototypische Kollokationen existieren. So ist es nicht in jedem Einzelfall unumstritten, ob man der jeweiligen Wortverbindung den Kollokationsstatus anerkennen sollte. Bei Zweifelsfällen lässt sich jedoch eine kontrastive Betrachtungsweise heranziehen. Wortverbindungen, deren Bestandteile nicht wörtliche Äquivalente in der L1 haben, werden auf jeden Fall als Kollokationen betrachtet. Sie als Einheiten zu vermitteln ist durchaus begründet, da sie enorm interferenzgefährdet sind. So lässt sich z. B. im folgenden Vorschlag

Trage in einem Kalender alle Prüfungstermine ein ...11

die Wortverbindung Termine eintragen aus ungarischer Perspektive auf jeden Fall als Kollokation betrachten, da die ung. Entsprechung des Kollokators auf Deutsch einschreiben (ung.: beir) heißt. Auf der Basis des kontrastiven Ansatzes kann der Kollokationsbereich sprachenpaarspezifisch abgesteckt werden. Die kontrastive Perspektive ist für den Lerner

\footnotetext{
${ }^{8}$ www.mytopic.at/home/schule-job/lerntipps/60, Stand 15.05.2010.

${ }^{9}$ www.mytopic.at/home/schule-job/lerntipps/60, Stand 15.05.2010.

10 wortschatz.uni-leipzig.delabfrage, Suche am 8. November 2010.

11 www.mytopic.at/home/schule-job/lerntipps/60, Stand 15.05.2010.
} 
relevant, da er in seinem mentalen Lexikon immer wieder auf die L1 als Stütze zurückgreift. Es ist jedoch unvorhersagbar, ob der Transfer gegebenenfalls positiv oder negativ ausfällt. Beim Vergleich kann eine weitere gelernte Fremdsprache herangezogen werden, wie das im Tertiärsprachenansatz durchgeführt wird.

Zusammenfassend lässt sich feststellen, dass Kollokationen als wortübergreifende Einheiten zu vermitteln und zu erlernen sind, wenn wir Kollokationen als Phraseme betrachten. Das Ziel ist dabei, eine psycholinguistische Festigkeit der jeweiligen Kollokation im mentalen Lexikon des Lerners zu entwickeln.

\section{$3 \quad$ Lehrwerke und Kollokation}

Der vorliegende Aufsatz stellt dar, dass Kollokationen in einigen linguistischen Disziplinen eingehend behandelt werden. Es wurde oben auch der Versuch unternommen, Wege aufzuzeigen, die die linguistischen Erkenntnisse für den Spracherwerbsprozess nützlich machen. Weitere wichtige Fragestellungen ergeben sich, wenn wir den Lerner in den Mittelpunkt der Überlegungen stellen. Das Ziel ist letztendlich, sein mentales Lexikon bezüglich Kollokationen $\mathrm{zu}$ formen. Wie ist jedoch die Einstellung der Lerner zu Kollokationen? Erkennen sie diese als feste Wortgruppen, die man als Einheiten betrachten sollte? Die Unterrichtspraxis zeigt, dass DaF-Lerner "wie alle Fremdsprachenlerner von Natur aus eher Word-Watcher [...] als Chunk-Sammler" sind (Handwerker/Madlener 2009: 6). Diese Aussage trifft auch auf die Kollokationen zu, wie einige empirische Studien belegen (cf. Reder 2008a; Reder 2008b). Dass Lerner von sich aus vorwiegend Einzelwörter als Wortschatzeinheiten erkennen, ist nicht weiter überraschend, wenn wir gängige Lehrmaterialien betrachten. Die meisten DaF-Lehrwerke begünstigen die "Word-WatcherEinstellung" der Lerner. Die Wortfixierung der Lehrwerke ist u.a. durch den Verzicht auf den Terminus gekennzeichnet. Mir ist kein überregionales DaF-Lehrwerk bekannt, das Lerner mit dem Kollokationsbegriff vertraut machen würde. Ein weiterer Nachteil ist, dass die meisten Lehrbücher in Glossaren vorwiegend Einzelwörter auflisten. Einige bringen unter dem Etikett "Ausdrücke" auch Idiome und kommunikative Formeln, aber nur vereinzelt Kollokationen.

Es ist jedoch den Lehrwerkautoren nicht ganz berechtigt vorzuwerfen, dass sie mit dem Terminus "Kollokation" kaum operieren und wenige Kollokationen in Glossaren anführen. Es gibt ja keine einheitliche Kollokationsauffassung in der Bezugswissenschaft, in der Linguistik. Die Kollokationsdeutungen in der Fremdsprachendidaktik, typische und übliche Wortverbindungen (cf. Hausmann 1984) oder Wortpaare, die kontextuell erwartbar sind (cf. Barkowski/Krumm 2010: 155), in der Praxis umzusetzen, ist auch nicht problemlos. Richten wir den Fokus auf den Lerner. Das Ziel ist, dass er in seinem mentalen Lexikon Kollokationen als Einheiten abspeichert. So ist es wohl für den Spracherwerb am effektivsten, wenn Kollokationen als phraseologische Einheiten erfasst werden. So plädiert vorliegender Aufsatz für die phraseologische Kollokationsdeutung und ihre Umsetzung in DaFLehrwerken. Dabei ist der kontrastive Ansatz nicht zu vernachlässigen, da der Lerner bereits in L1 gespeicherte Kollokationen in seinem mentalen Lexikon präsent hat. In der Ausgangsund in der Zielsprache benötigen nichtäquivalente Kollokationen vermutlich mehr Training. So können regionale Lehr- und Ergänzungsmaterialien effektiv eingesetzt werden. Sie haben nämlich den Vorteil, dass sie in der Lage sind, ihre spezifische Zielgruppe und so die Erstsprache der Lerner zu berücksichtigen. Sie können Übungen anbieten, in denen unter kontrastivem Ansatz ausgewählte Kollokationen geschult werden. Es empfiehlt sich für die Kollokationsvermittlung die Anwendung des Drei-Phasen-Modells. Die Umsetzung dieses Modells finden wir z. B. im "Übungsbuch zur Schulung sprachlicher Kompetenzen" (Reder / Jaszenovics 2010). Ein exemplarisches Beispiel ist die kontextuelle Vermittlung der Kollokationen Punkte (miteinander) verbinden und Stift absetzen. Diese Kollokationen werden in einem Impulstext dargeboten und von den Lernern entdeckt sowie mit den L1- 
Äquivalenten verglichen. Der Gebrauch dieser Kollokationen wird in inhaltsorientierten Sätzen eingeübt. Der Übungsphase schließt sich eine gelenkte Textproduktion an. Der Lernprozess endet mit der Besprechung von Lernstrategien zu Kollokationen (cf. Reder 2010: 76-83; 124f.).

Ein wichtiger Aspekt ist bei der Auswahl von Übungstypen, dass sich gut bekannte, traditionelle Aufgaben kontraproduktiv auf die Kollokationsschulung auswirken können. Nehmen wir zur Veranschaulichung dieses Phänomens einen Impulstext über Lernstrategien, den wir mit einer Lernergruppe behandeln ${ }^{12}$. Wie ist die folgende Aufgabenstellung in Bezug auf die Kollokationsschulung zu bewerten? Fasst den Text mit euren eigenen Worten zusammen. Wenn das Ziel ist, dass sich die Lerner unbekannte Kollokation aneignen sollen, dann wäre es effektiver nicht mit den eigenen Worten den Text wiederzugeben. Schlüsselwörter und die neuen Kollokationen sollten zur Inhaltsangabe vom Lerner selbst aus dem Text extrahiert oder durch den Lehrer bereitgestellt werden. Das Ziel ist, nicht diese zu vermeiden, sondern zu verwenden. Eine der Inhaltsangabe vorangehende Aktivität könnte mit der folgenden Aufgabenstellung angeregt werden:

Gib einem Freund Vorschläge, wie man erfolgreich lernen kann. Sammle dazu im Text Wörter und Kollokationen (Wörter mit ihren festen Partnern).

Mit solchen und ähnlichen Übungen kommen Kollokationen vermutlich auch unter Lernern in Mode.

\section{Literatur}

Barkowski, Hans/Krumm, Hans-Jürgen (eds.) (2010): Fachlexikon Deutsch als Fremd- und Zweitsprache.Tübingen und Basel: A. Franke/UTB.

Burger, Harald (2007): Phraseologie. Eine Einführung am Beispiel des Deutschen. Berlin: Schmidt (= Grundlagen der Germanistik 36).

Bubenhofer, Noah/Ptashnyk, Stefaniya (2010): "Korpora, Datenbanken und das Web: State of the Art computergestützter Forschung in der Phraseologie und Lexikographie". In: Ptashnyk, Stefaniya/Hallsteinsdóttir, Erla/Bubenhofer, Noah (eds.): Korpora, Web und Datenbanken: Computergestützte Methoden in der modernen Phraseologie und Lexikographie. Baltmannsweiler, Schneider Verlag Hohengehren: 7-19.

Donalies, Elke (2009): Basiswissen. Deutsche Phraseologie. Tübingen und Basel: A. Franke/UTB.

Dörr, Simone (2005): Vom WWW zur Kollokation. Trier: Wissenschaftlicher Verlag.

Handwerker, Brigitte/Madlener, Karin (2009): Chunks für DaF. Theoretischer Hintergrund und Prototyp einer multimedialen Lernumgebung (inklusive DVD). Baltmannsweiler: Schneider Hohengehren.

Hausmann, Franz Josef (1984): Wortschatzlernen ist Kollokationslernen. Praxis des neusprachlichen Unterrichts 31: 395-406.

Hausmann, Franz Josef (2003): "Was sind eigentlich Kollokationen?" In: Steyer, Kathrin (ed.): Wortverbindungen - mehr oder weniger fest. Berlin, de Gruyter: 309-335.

Hausmann, Franz Josef (2007): Kollokationen im Rahmen der Phraseologie. Systematische und historische Darstellung. Zeitschrift für Anglistik und Amerikanistik 55: 217-234.

Hollós, Zita (2004): Lernerlexikographie: syntagmatisch. Konzeption für ein deutschungarisches Lernerwörterbuch. Tübingen: Niemeyer. (= Lexikographica Serios Maior 116).

Lütge, Christiane (2002): Syntagmen und Fremdsprachenerwerb. Frankfurt: Lang.

Konecny. Christina (2010): "Lexikalische Kollokationen und der Beitrag der InternetSuchmaschine Google zu ihrer Erschließung und Beschreibung". In: Ptashnyk,

12 www.mytopic.at/home/schule-job/lerntipps/60, Stand 15.05.2010. 
Stefaniya/Hallsteinsdóttir, Erla/Bubenhofer, Noah (eds.): Korpora, Web und Datenbanken: Computergestützte Methoden in der modernen Phraseologie und Lexikographie. Baltmannsweiler, Schneider Verlag Hohengehren: 77-95.

Reder, Anna (2006): "Kollokationsforschung und Kollokationsdidaktik". Linguistik online. 28/3/06: 157-176. www.linguistik-online.de/28_06/reder.pdf, Stand 8. November 2010.

Reder, Anna (2008a): "Erkennen DaF-Lernende Kollokationen?" In: Scheibl, György (ed.): Tests im DaF-Unterricht - DaF-Unterricht im Test. Szeged, Grimm: 105-115.

Reder, Anna (2008b): "Eine Stunde halten - Kollokationen in der Schullexik". In: Canisius, Peter /Hammer, Erika (eds.): 50 Jahre Germanistik in Pécs. Wien, Praesens: 159-179.

Reder, Anna (2010): "Geometrische Figuren". In: Reder Anna/Jaszenovics Sándor (eds.): Cool-Tour-Hauptstädte. Übungsbuch zur Schulung sprachlicher Kompetenzen im Deutschunterricht. Pécs, Universität Pécs: 76-83, 124-125.

Reder Anna/Jaszenovics Sándor (eds.) (2010): Cool-Tour-Hauptstädte. Übungsbuch zur Schulung sprachlicher Kompetenzen im Deutschunterricht. Pécs: Universität Pécs. http://bit.ly/fNedjv (gesehen am 12.09.2011).

Scherfer, Peter (2001): "Zu einigen wesentlichen Merkmalen lexikalischer Kollokationen". In: Lüger, Heinz-Helmut/Lorenz-Bourjot, Martin (eds.): Phraseologie und Phraseodidaktik. Wien, Edition Praesens: 3-21.

Schönefeld, Doris (2001): Where Lexicon and Syntax meet. Berlin: de Gruyter (= Trends in Linguistics, Studies and Monographs 135).

Siepmann, Dirk (2003): Kollokationen und Fremdsprachenlernen. Vortrag an der Universität Stuttgart am $10 . \quad$ November 2010. stuttgart.de/lingrom/lehre/fortbildung2003/siepmann-vortrag.pdf, Stand 20.05. 2010.

Steinbügel, Birgit (2005): Deutsch-englische Kollokationen. Erfassung in zweisprachigen Wörterbüchern und Grenzen der korpusbasierten Analyse. Tübingen: Niemeyer. 